\title{
The detection of subtelomeric chromosomal rearrangements in idiopathic mental retardation
}

\author{
Jonathan Flint ${ }^{1}$, Andrew O.M. Wilkie ${ }^{1}$, Ve oni a J. Buckle ${ }^{1}$, Robin M. Winter ${ }^{2}$, \\ Anthony J. Holland ${ }^{3}$ \& Heather E. McDerı lid $^{4}$
}

\begin{abstract}
A major challenge for human genetics is to identify new causes of mental retardation, which, although present in about $3 \%$ of individuals, is unexplained in more than half of all cases. We have developed a strategy to screen for the abnormal inheritance of subtelomeric DNA polymorphisms in individuals with mental retardation and have detected three abnormalities in 99 patients with normal routine karyotypes. Pulsed-field gel electrophoresis and reverse chromosome painting showed that one case arose from an interstitial or terminal deletion and two from the de novo inheritance of derivative translocation chromosomes. At least $6 \%$ of unexplained mental retardation is accounted for by these relatively small chromosomal abnormalities, which will be an important resource in the characterization of the genetic basis of neurodevelopment.
\end{abstract}

\begin{abstract}
'Institute of
Molecular

Medicine, John

Radcliffe Hospital,

Oxford OX3 9DU,

UK

${ }^{2}$ Mothercare Unit of

Paediatric Genetics and Fetal Medicine,

Institute of Child

Health, London,

WCIN $1 E H, U K$

${ }^{3}$ Department of

Psychiatry,

Developmental

Psychiatry Section,

University of

Cambridge,

Cambridge, CB2

$2 A H, U K$

${ }^{4}$ Department of

Genetics, University

of Alberta,

Edmonton, Canada

T6G 2E9

Correspondence should be addressed to J.F.
\end{abstract}

Mental retardation is a common and distressing disorder
whose origins are poorly understood. About three percent
of the population have an intelligence quotient (IQ) less
than 70 (refs 1,2 ) yet a cause for mental retardation is
established in less than half of all cases ${ }^{3-15}$. Despite recent
advances in understanding the molecular basis of some
known causes of mental retardation, progress in
unravelling idiopathic mental retardation has been slow,
partly because of the aetiological heterogeneity of the
condition. The large number of known conditions that
result in mental retardation is probably matched by an
equally large number of unknown conditions.

Routine cytogenetic analysis indicates that chromosomal anomalies constitute $40 \%$ of severe (IQ < 55 ) and $10-20 \%$ of mild mental retardation (IQ 55-70); however, rearrangements involving less than 1 or 2 megabases $(\mathrm{Mb})$ are undetectable even at the highest resolution and could account for a substantial number of idiopathic cases. Hypervariable DNA polymorphisms (HVPs) detected by probes for variable number of tandem repeats (VNTR) can be used to find such cryptic chromosomal abnormalities by the observation of abnormal inheritance of alleles from the parents ${ }^{16}$. By the use of this method, deletions, duplications and uniparental disomy (UPD) can be detected, although a prohibitively large number of loci would need to be screened to cover the whole genome (for example, 3,000 at 1-Mb intervals). Consequently a search for cryptic chromosomal abnormalities needs to be more focused.

There are indications that the terminal regions of chromosomes may be enriched for cryptic chromosomal abnormalities. Cytogenetically invisible unbalanced translocations of chromosome ends have recently been shown to be the cause of mental retardation in the $\alpha$-thalassaemia mental retardation syndrome (ATR16) ${ }^{17,18}$, Wolf-Hirschhorn syndrome ${ }^{19,20}$, Miller-Dieker syndrome ${ }^{21}$ and cri-du-chat syndrome ${ }^{22}$. In a second type of terminal rearrangement, a chromosome is truncated and stabilized by the addition of telomeric repetitive DNA. One case of the ATR-16 syndrome due to a telomeric truncation has been characterized at a molecular level by DNA sequence analysis ${ }^{23}$, and the process probably accounts for many other terminal deletions.

HVP analysis will also detect UPD, which has been implicated as a cause of retardation in Prader-Willi, Angelman, and Beckwith-Wiedemann syndromes ${ }^{24,25}$. A few highly polymorphic markers from any part of the chromosome will suffice to identify UPD affecting the whole chromosome, but the use of telomeric markers gives a higher chance of picking up segmental UPD: mitotic recombination due to a single crossover giving rise to segmental UPD will always involve the telomere.

In this study, the subtelomeric regions of 28 chromosome ends were analysed in 99 mentally retarded individuals with highly polymorphic probes. Three cases of monosomy were identified (two involving $22 \mathrm{q}$ and one $13 q$ ), and confirmed by fluorescence in situ hybridization (FISH), or densitometric analysis. Reverse chromosome painting demonstrated that two cases were the unbalanced products of translocation; pulsed-field gel electrophoresis (PFGE) showed that the third case arose from a de novo interstitial or terminal deletion. We found no abnormalities at either the $22 \mathrm{q}$ or $13 \mathrm{q}$ locus in control populations ${ }^{26-28}$. This study is the first demonstration that cryptic chromosomal abnormalities make a significant contribution to idiopathic mental retardation. 
The cases described here, and others that can beidentified using a similar screening strategy, provide a starting point for the isolation and characterization of dosage-sensitive genes involved in neurodevelopment.

Detection of cryptic chromosomal rearrangements A sample of 99 individuals (58 males and 41 females) with idiopathic mental retardation ranging from mildly to severely affected were identified. In all cases chromosome abnormalities were excluded by routine cytogenetic analysis (400-500 band level). Affected individuals could be divided, on clinical grounds, into two groups: 68 with physical stigmata suggestive of an underlying chromosomal disorder, and 31 with no such stigmata. Clinical signs of a chromosomal disorder were one or more congenital anomalies (including dysmorphic features) in the absence of a recognizable syndrome.

A total of 28 chromosome tips were surveyed covering 23 different chromosomes (Table 1). Only chromosome 1 was not surveyed at either telomere. HVP analysis suggested a chromosomal abnormality in two cases from the first group of affected individuals, and in one case from the second. The abnormalities were then confirmed by FISH or densitometric analysis.

\section{Case 1}

Patient AH is a 3-year-old boy with developmental delay, mild facial dysmorphism and microcephaly. Family history is negative for mental retardation. Early motor milestones were within normal limits, but by age 26 months he was functioning at the 18 month level.

Southernblot analysis of $D 135107$ (probe CEB5localized to the terminal band of $13 \mathrm{q}$ (ref. 27) showed failure to inherit a paternal allele (Fig. 1). The same result was obtained using three different endonucleases, thus excluding a size difference due to a mutation in an endonuclease recognition site. Information from at least 20 probes confirmed inheritence of alleles from both parents at other loci. A second VNTR from terminal 13q, D13S103 (probe MS626 (ref. 29) also detected an abnormality.

High resolution prometaphase karyotype analysis was normal, but FISH confirmed the deletion. A cosmid containing the $D 13 S 107$ locus hybridized to one $13 q$ homologue only in 50 metaphases examined (Fig. $2 a$ ), although no such abnormality was found in the child's parents. FISH using the plasmid MS626 showed that this locus too was deleted in $\mathrm{AH}$ but not in his parents (not illustrated). Thus $\mathrm{AH}$ has a de novo deletion in the subtelomeric region of chromosome 13q.

\section{Case 2}

Patient LM is a 22-year-old female with severe mental retardation (IQless than 20) and marked facial dysmorphism. Family history is negative for mental retardation. She has nolanguage, but is fully mobile. Her facial appearance is markedlyabnormal withmicrognathia, simple ears, prominent nose and a gap between her upper two incisors. In addition she has long thin fingers, $2 / 3$ syndactyly of the feet (also present in other unaffected family members), a right talipes equinovarus with a pes cavus and a left calcaneovalgus deformity.

Southern blot analysis showed lack of a maternal allele at D22S163 (probe MS607 on chromosome 22q) (Fig. 1), a result found with three different endonucleases. FISH analysis of D22S163 was carried out with four cosmids, isolated from a chromosome 22 library by screening with MS607 (Jan Dumanski, unpublished results). All detected a deletion of $22 q$ in the case of LM (Fig. $2 b$ ), although her parents were normal. Thus she has a de novo deletion in the subtelomeric region of chromosome 22q.

Restriction fragment length polymorphism (RFLP) and densitometric analysis were performed to 


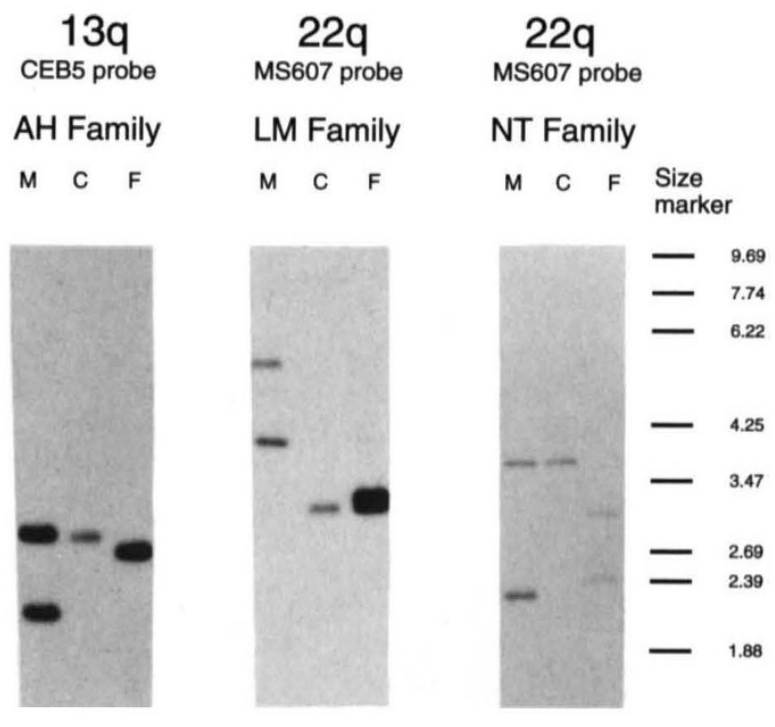

examine the extent of the deletion in LM (Fig. 3). These investigations showed that the breakpoint occurs between D22S97 and D22S22/D22S94 and encompasses approximately 25 centimorgans of the most telomeric region of $22 \mathrm{q}$.

Although routine karyotyping had been reported normal, repeat analysis at high resolution suggested that there was an anomalous chromosome 22 . The banding pattern was not distinctive enough to allow definitive characterization: it was consistent with a de novoderivative translocation, a paracentric inversion or a more complex rearrangement.

\section{Case 3}

Patient NT is a 12-year-old boy with mild mental retardation (IQ 64), normal physical features and a family history negative for mental retardation. The neonatal period and early motor milestones were within normal limits. He presented aged 2.5 years with no speech and made his first definable words aged four years. At 12.5 years his particular speech difficulties persist, and he has a 25-point discrepancy between verbal IQ (50) and performance IQ (75). He has no abnormal physical findings or dysmorphic features apart from bilateral accessory nipples.

Like the previously described patient, NT had an abnormality initially detected by analysing D22S163 (using probe MS607) (Fig. 1). The HVP analysis showed absence of a paternally inherited allele, and again non-mendelian inheritence at other loci and mutation in the endonuclease recognition site were excluded. Densitometric analysis was used to compare NT to a normal control and a known $22 \mathrm{q}$ deletion in five replicate samples. The signals from NT were significantly less than those from the normal individual and not significantly different from the known $22 q$ deletion, demonstrating that one copy of MS607 is deleted in NT (data not shown). The same technique showed that probes mapping proximal to MS607 were not deleted (Fig. 3).

His high-resolution karyotype was normal, but, surprisingly, FISH analysis with the same four 22q cosmids used in the previous case revealed a signal on both copies of chromosome 22 (not shown). A possible explanation for the discrepancy between the densitometric and FISH
Fig. 1 Molecular detection of subtelomeric rearrangements in three patients with mental retardation. Three Southern blots are illustrated with samples from the affected child (C) flanked by mother $(M)$ and father $(F)$. The sizes of bands in kilobases are shown on the right. In the $\mathrm{AH}$ family, the Southern blot demonstrates the failure to inherit an allele from the father at D13S107 (CEB5 probe) on chromosome 13q. In the LM family, autoradiography with probe MS607 (D22S163) from chromosome 22q shows failure to inherit a maternal allele, and in the NT family the same probe shows failure to inherit a paternal allele.

analyses was that a breakpoint occurred within the region detected by the cosmids. Accordingly a probe (HE 7.0) free of highly repetitive DNA was isolated from one cosmid and used to map the locus in more detail. Digested genomic DNA from the parents and child was separated by PFGE and probed with HE 7.0. Five enzymes (NotI, MluI, NruI, Sfil and SstII) gave a similar pattern: a single band in the parents, and two in the child (Fig. 4), demonstrating that a de novo rearrangement is present in the child. With all five enzymes the breakpoint fragment in NT was shorter than the normal by about 60 kilobases $(\mathrm{kb})$, making it very likely that NT has a $60 \mathrm{~kb}$ interstitial or terminal deletion rather than a translocation.

\section{Reverse chromosome painting}

We used reverse chromosome painting to investigate further the nature of these rearrangements. By labelling the abnormal chromosomes and hybridizing them to normal metaphases, any translocated material can be detected as an additional signal on a second pair of chromosome homologues. All flow karyotypes were normal and, as in no case could the abnormal chromosomes be distinguished from their normal homologues, both chromosomes were labelled together.

The sorted and labelled chromosome 13 s from patient $\mathrm{AH}$ detected a signal on Xp and $\mathrm{Yp}$ that was not seen in a control experiment using a normal chromosome 13 (Fig. 5). We confirmed this finding by using a biotinylated cosmid from the pseudoautosomal region of Xp (CY29 (ref. 30)). When hybridized to AH's chromosomes, the cosmid detected a signal on the end of one 13q homologue in addition to signals from the pseudoautosomal region on $\mathrm{Xp}$ and $\mathrm{Yp}$ (Fig. 6). Thus $\mathrm{AH}$ is trisomic for the terminal region of $\mathrm{Xp} / \mathrm{Yp}$ and deleted for the terminal region of $13 \mathrm{q}$.

Reverse paints prepared from the sorted chromosome 22 s of patient LM consistently revealed an extra signal on $9 q$ (Fig. 7). This signal was observed on all metaphases from three different normal males, and no similar signal was seen with a control experiment. Because of a lack of suitable subtelomeric 9q probes, we have not been able to characterize this trisomy further, but the results indicate that the patient has a de novo unbalanced chromosome derived from a translocation involving the terminal regions of $22 \mathrm{q}$ and $9 \mathrm{q}$, which has resulted in a deletion of subtelomeric $22 \mathrm{q}$ and a trisomy of subtelomeric $9 \mathrm{q}$.

In the case of NT, hybridization to male metaphase chromosome preparations revealed no additional chromosomal material to that observed in a control experiment using sorted and labelled normal chromosome 22 (not illustrated). This experiment supports the inference from PFGE that NT has a true deletion rather than a translocation of 22q. 

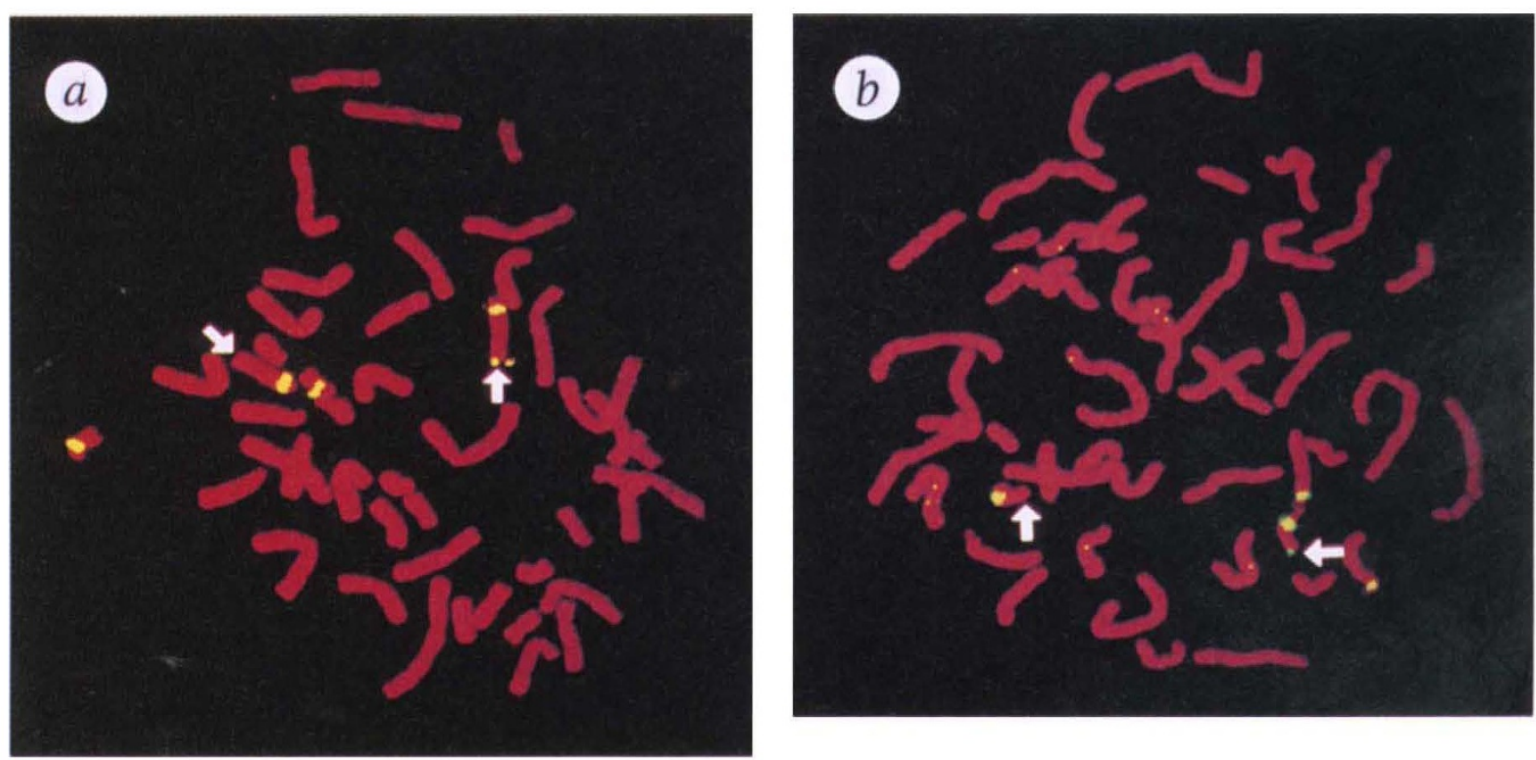

Fig. 2 In situ hybridization and fluorescent detection. a, Metaphase chromosomes of patient AH. Arrows indicate the telomeres on the long arm of the chromosome 13 pair, which have been identified by an $\alpha$-satellite repeat probe (yellow signal at the centromere). Signal from biotinylated cosmid CEB5 is absent from one chromosome 13q telomere, demonstrating a deletion. The satellite repeat also identifies the chromosome 21 pair because of cross-hybridization between the centromeric sequences. $b$, Metaphase chromosomes of patient LM. Arrows indicate the telomeres on the long arm of the chromosome 22 pair, this time identified by $\alpha$-satellite repeats that cross-hybridize to chromosome 14 . The single hybridization signal of a cosmid containing MS607 demonstrates the deletion of one 22q subtelomeric locus.

\section{No rearrangements at $22 q$ and $13 q$ in controls}

In order to exclude the possibility that terminal deletions of chromosomes $13 q$ and $22 q$ are relatively common rearrangements with little or no associated phenotype, we examined 240 nuclear families by Southern analysis using the probes MS607 and CEB5. The sample included 117 European, three Filipino and 60 Asian families. No family included members with mental retardation. In the samples, 186 families were informative for the presence of deletions in the case of probe MS607 and 160 families for probe CEB5. No deletions were detected. The 95\% confidence limits for deletions at MS607 and CEB5 in a population without mental retardation are $0-1.6 \%$ and $0-1.9 \%$, respectively.

If $22 \mathrm{q}$ and $13 \mathrm{q}$ translocations were relatively common, we might expect to discover the reciprocal products (that is trisomies for $22 q$ and $13 q$ ). As part of a larger study to investigate the distribution of VNTR alleles in different world populations we have examined 3,000 unrelated individuals with the probes MS607 and MS626 (the other $13 \mathrm{q}$ probe deleted in patient $\mathrm{AH}$ ). In no case were three alleles seen in over 2,000 informative analyses for each locus. This suggests that trisomies at these loci are rare.

Taken together with the published data for these loci ( 700 meioses in $\mathrm{CEPH}$ pedigrees with the 13q probe and 80 meioses with the $22 q$ probe $^{26-28}$ ), we conclude that our data are inconsistent (at a 0.05 significance level) with a prevalence of $22 \mathrm{q}$ deletions as great as $1 \%$ and with a prevalence of $13 \mathrm{q}$ deletions as great as $0.5 \%$.

\section{Informativeness of VNTRs}

In addition to detecting three cryptic chromosomal abnormalities, this survey provided an opportunity to assess the effectiveness of the screening strategy and the sensitivity of the test. The latter information can then be used to estimate the prevalence of cryptic chromosomal abnormalities in the population surveyed. Direct measures of informativeness for the detection of monosomy,
Fig. 3 Restriction fragment and densitometric analysis of two patients with 22q deletions. On the left is a diagram of the terminal region of 22q showing the recombination fractions $(\theta)$ between the loci examined. The location of D22S163 near ARSA is based on PFGE data (unpublished). ND, not done.

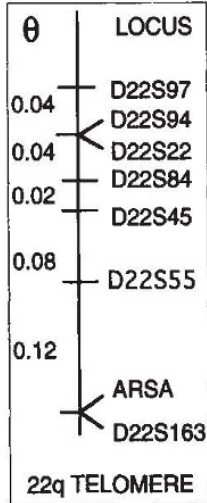

\begin{tabular}{|c|c|c|c|c|c|}
\hline & Locus & Patient LM & & Patient NT & Method of analysis \\
\hline $\mathrm{KI}-260$ & D22S97 & Not deleted & densltometric & $\mathrm{ND}$ & \\
\hline KI-1105 & D22S94 & Deleted & densitometric & ND & \\
\hline W110D & D22S22 & Deleted & densitometric & Not deleted & PFP \\
\hline $\mathrm{KI}-216$ & D22S84 & Maternal deletion & FFP & ND & \\
\hline H41a & D22S45 & Matemal deletion & PFP & & RFLP uninformative \\
\hline pH91 & D22S55 & & & Not deleted & densitometric \\
\hline & ARSA & Maternal deletlon & FPP & Not deleted & densitometrlc \\
\hline AS607 & D22S163 & |Matemal deletion & RPP & |Patemal deletion & RFLP, densitometric \\
\hline
\end{tabular}




\section{$22 q$}

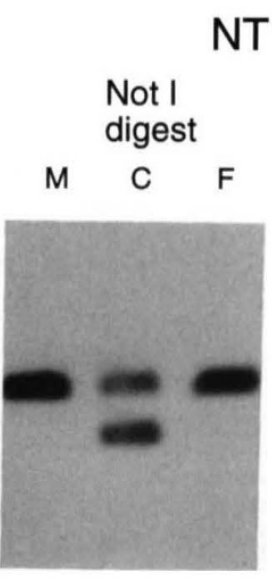

\section{NT Family}

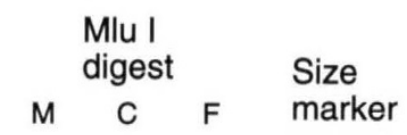

Fig. 4 Southern blot of a pulsed-field gel probed with fragment HE 7.0. Two different digests (Notl and Mlul) are shown of samples from the NT family: M, mother, $\mathrm{C}$, child and $\mathrm{F}$, father. A size marker in kilobases is shown on the right.

trisomy, hetero- and isodisomy derived from the distribution of parental mating types are shown in Table 2. In the majority of cases the interpretation of results was straightforward. Small alleles not readily detected by blotting made analysis difficult in a few instances. VNTR mutations were detected in 31 cases (Table 2), mostly at D2S90, which has a mutation rate of approximately $15 \%$ (ref. 27).

Prevalence of cryptic subtelomeric abnormalities Of 99 children with idiopathic mental retardation, three were found to have cryptic chromosomal abnormalities. The observed frequency is therefore $3 \%$, but this estimate must be corrected for the fact that only 28 telomeres were surveyed and that in many cases the mating types were not informative. Once this is taken into account, the chance of

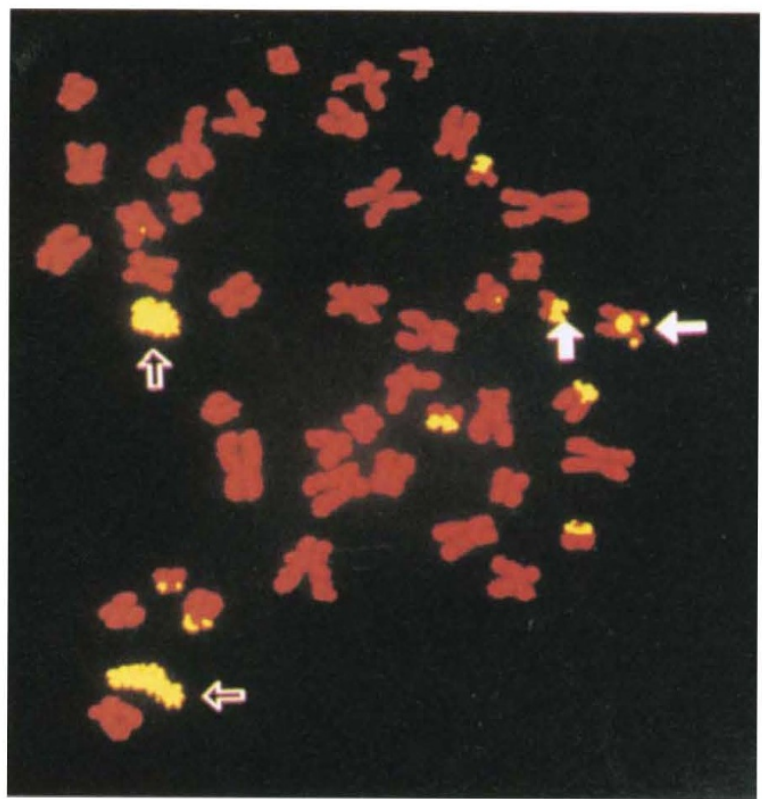

detecting a random abnormality is $52 \%$ for subtelomeric monosomy and $69 \%$ for uniparental disomy ${ }^{16}$. Assuming that every telomere has the same probability of undergoing rearrangement (and therefore that the 28 chromosome tips surveyed are representative) the best estimate of the frequency of cryptic subtelomeric monosomy in unexplained mental retardation is $5.8 \%$ ( $95 \%$ confidence limits are 1.2-17.6\%). No instances of UPD were detected in this survey, and the $95 \%$ confidence limits for the frequency of UPD are $0-4.4 \%$.

\section{Discussion}

The results presented here show for the first time how a combination of HVP analysis, FISH, PFGE and reverse chromosome painting can be used to screen for and characterize cryptic chromosomal rearrangements. These rearrangements are very likely to cause mental retardation in individuals in which they occur: all of the probes used in this study have been employed in linkage mapping in normal individual ${ }^{28}$, and no cases of de novo deletion or UPD have been described in more than 11,000 informative meioses (since 35 probes were examined, this figure is approximately equivalent to investigating 300 nuclear families). In all instances where abnormal inheritance patterns have been found, they have been due to mutations at the VNTR loci or to the occurrence of small VNTR alleles undetectable by Southern blotting ${ }^{27,31,32}$.In addition, investigation of 240 nuclear families and of 3,000 unrelated individuals with the $13 q$ and $22 q$ probes that detected deletions in our patient sample revealed no rearrangements (either deletions or trisomies). However, characterization of the rearrangements' effects on gene expression is needed to prove that they cause mental retardation.

Cryptic chromosomal abnormalities have often been suspected to contribute to the pathogenesis of idiopathic retardation, but there have been no attempts to assess the magnitude of that contribution. The data presented here allow a preliminary assessment: three de novo deletions were found in 99 individuals surveyed using a test which would detect $52 \%$ of such abnormalities. This gives a best estimate of about $6 \%$ for the frequency of cryptic subtelomeric rearrangements. However, this figure must be conservative as many of the probes used in this study may be several megabases from their cognate telomere; in these cases analysis will have failed to detect small terminal rearrangements.

No cases of UPD were discovered. Because the test was informative for $69 \%$ of such cases, the result excludes UPD as contributing to more than $5 \%$ of idiopathic mental retardation with $95 \%$ confidence, suggesting that UPD is a relatively rare occurrence ${ }^{33}$. There was no difference in frequency of monosomy between the clinical groups with $(2 / 68)$, or without $(1 / 31)$, physical stigmata of a chromosomal abnormality. A more complete survey is needed to determine the correlation of subtelomeric

Fig. 5 Reverse chromosome paint of chromosome 13 from patient AH onto a normal male metaphase. Outlined arrows mark the painted chromosome 13s. Signal is seen on acrocentric short arms (as found in a control), but there is also a signal on the short arm of the $X$ and $Y$

chromosomes. A centromere marker identifies the $X$ chromosome, and filled arrows indicate the signal on $X p$ and $Y p$. 
abnormalities with physical stigmata and the frequency of UPD in mental retardation.

Our results support the view that chromosomal rearrangements affect subtelomeric regions more often than other parts of the genome. Twenty-eight telomeres were examined, and in $76 \%$ of cases the analysis was informative for monosomy, so the equivalent of 21 telomeres were fully analysed. We can expect to have excluded deletions up to $5 \mathrm{Mb}$ (the maximum resolution of routine cytogenetic screening), but may have failed to detect those affecting only the last megabase of the chromosome (assuming that most of the probes used will be more than a megabase from the telomere). Thus in assaying 99 children we have surveyed approximately $8,300 \mathrm{Mb}$ and found three deletions, or about one deletion every $3,000 \mathrm{Mb}$. Since the haploid genome is only 3,000 $\mathrm{Mb}$, this suggests either that all the remaining cases of idiopathic mental retardation in this survey have deletions

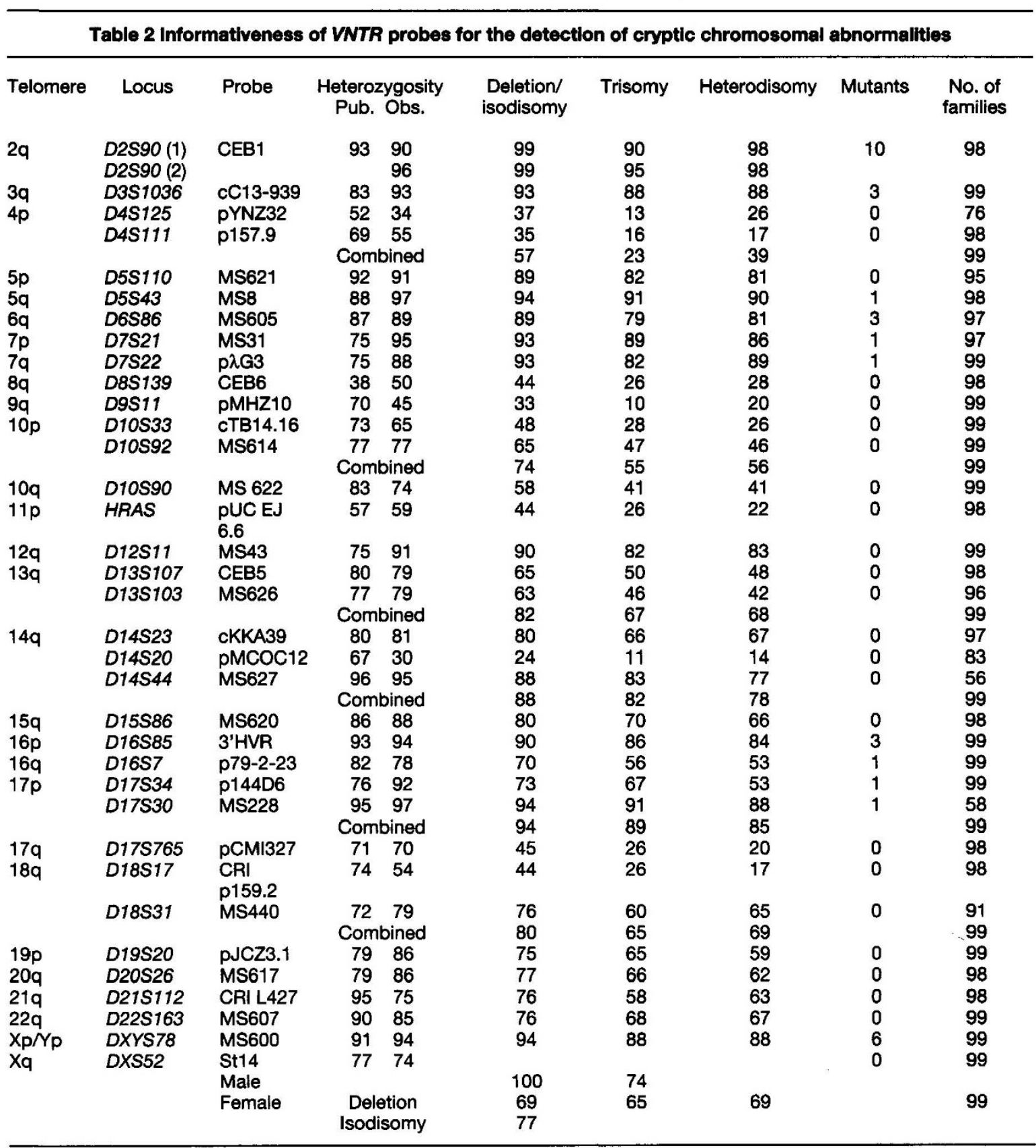

The published heterozygosity (Pub.) is compared with the observed (Obs.). Observed heterozygosity was calculated from the parental genotypes. In the case of D2S90 two figures are given, because null alieles may occur. In the first (D2S90 (1)), all parents with a single band on autoradiography are called homozygotes. In the second (D2S90 (2)), another VNTR in the cosmid CEB1 is used to to infer the presence at D2S90 of an allele undetectable by Southern blotting. Note that the distribution of mating types determines the informativeness of each probe ${ }^{16}$. Heterozygosity only provides an indirect estimate of informativeness. Moreover, sex-linked probes (such as St14) differ in informativeness between males and females. In males, deletions are always detectable and UPD cannot occur; in females, UPD can only arise from the mother and this gives differing detection rates for deletions and isodisomy. 


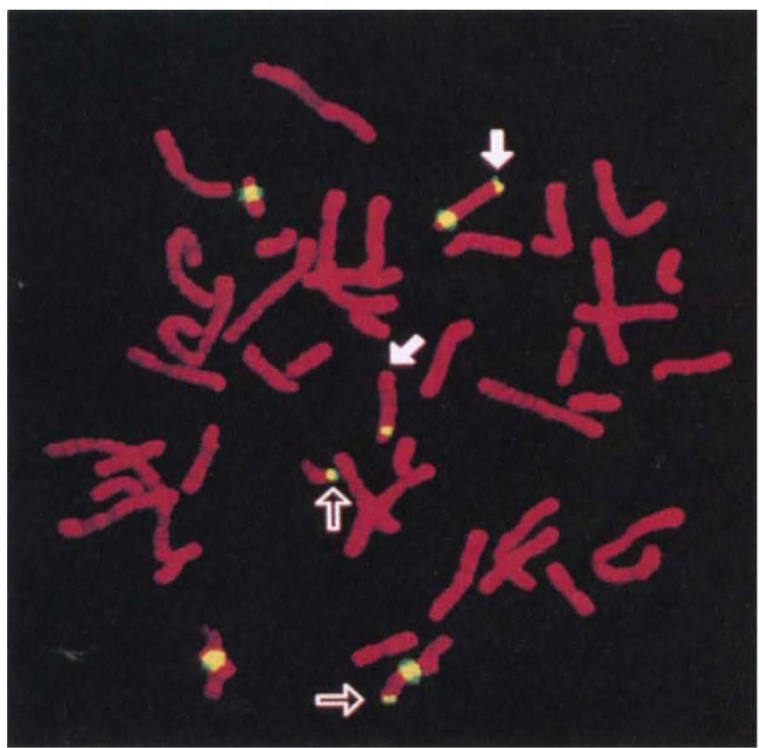

Fig. 6 FISH of pseudoautosomal cosmid CY29 onto AH metaphase preparations. Centromere markers identify chromosomes $X, 13$ and 21 . Telomeric signal on the sex chromosomes is indicated by outlined arrows, and filled arrows indicate the $13 \mathrm{q}$ telomeres, one of which carries a signal attributable to CY29 hybridization.

somewhere in their genomes, or that the frequency of deletions is relatively increased near the ends of chromosomes. Currently, the latter conclusion is the more reasonable.

This study demonstrates that HVP analysis is a useful investigation into the cause of developmental delay and mental retardation. Characterization of telomeric regions has alreadybegun to yield microsatellites physically linked to telomeres ${ }^{34}$. These HVPs can be screened quickly, and by using two or three microsatellites close to each telomere

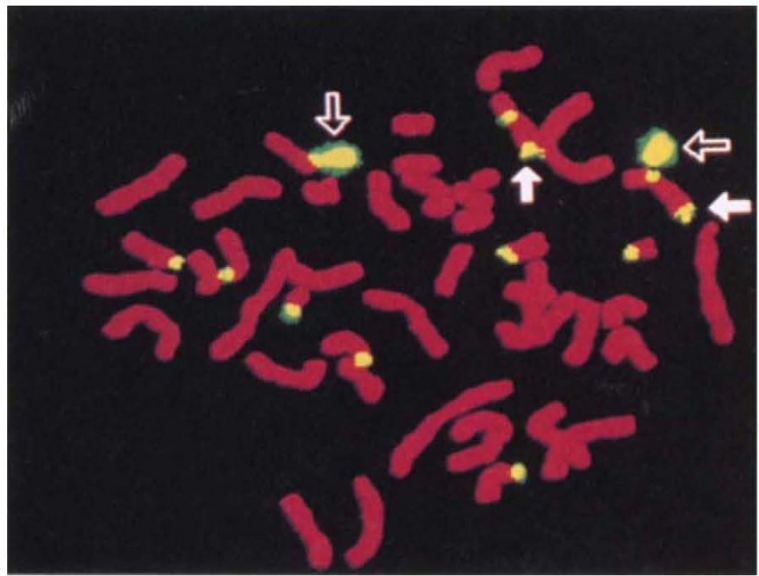

Fig. 7 Reverse chromosome paint of chromosome 22 from patient LM onto a normal male metaphase. Outlined arrows mark the chromosome 22 pair. Signal is seen on the short arms of the acrocentric chromosomes (as found in controis), but in addition a signal is present on the long arm of chromosome 9 (marked by filled arrows). The chromosome 9 pair is identified by a centromere-specific marker. the test could become virtually $100 \%$ informative. Although other methods of genomicscanning are available, such as prometaphase banding and $\mathrm{FISH}^{35}$, only HVP analysis can attain high sensitivity and specificity for the detection of both UPD and structural chromosomal rearrangements. Moreover, it is the technique with the greatest potential for automation ${ }^{36}$.

Finally, it is important to consider the implications that the discovery of small chromosomal aberrations may have for understanding the relationship between genetic lesion and impaired cognitive function. Small rearrangements with specific effects have proved invaluable in the past in the isolation of disease loci. The case of NT is the most interesting in this respect. The PFGE experiment suggests that the deletion may be as small as $60 \mathrm{~kb}$. If so, then its effects could indeed be very specific, and characterization of the affected region will be relatively straightforward. It may provide an opportunity to access dosage-sensitive genes involved in central nervous system development and function.

\section{Methodology}

Patient selection. A sample of 58 males and 41 females with idiopathic mental retardation was identified by examining the case records of a tertiary referral clinical genetics unit and of two community learning disability teams in south London. Patients were selected on the basis of (i) an IQ less than 70 or, if too young for psychological assessment, the presence of significant developmental delay; (ii) no recognizable syndrome whether chromosomal, Mendelian, or environmental; (iii) both parents available and willing to provide a blood sample. Consanguinity was not an exclusion criterion, but only one child was the offspring of a consanguineous mating (first cousin marriage).

Cytogenetic reports of all cases were reviewed. Where no recent analysis had been performed, the patient's chromosomes were examined at the $400-500$ band level. FragileX syndrome was excluded both by culturing cells in folate-deficient medium and by molecular analysis. In the latter case DNA was digested with Bgll endonuclease, blotted onto a nylon membrane and probed with a FRAXA clone Ox1.9 (ref. 37).

Control samples were made available to us from ongoing investigations into families with $\beta$-thalassaemia or with hypertension and families and individuals collected during surveys of genetic diversity in different world populations.

Probecollection. Three requirements governed the choice of probes: high heterozygosity, subtelomeric location and the production of a pattern on autoradiography from which the inheritance of all alleles could be determined unambiguously. Theoretical analysis shows that the informativeness of probes drops sharply with decrease in heterozygosity. Probes with a heterozygosity of $80 \%$ will detect only $50 \%$ of cases of trisomy and uniparental heterodisomy (see section below on calculation of informativeness) ${ }^{16}$. Thirty-five VNTRs with high heterozygosity and localization to the terminal band of a chromosome were selected for this study (Table 1). Only four probes are physically localized with respect to their cognate telomere: $\alpha$ globin $3^{\prime} \mathrm{HVR}$ which is $170 \mathrm{~kb}$ from the $16 \mathrm{p}$ telomere ${ }^{38}$; p157.9 and pYNZ32, $1.6 \mathrm{Mb}$ and $2.2 \mathrm{Mb}$, respectively, from the end of $4 \mathrm{p}^{39,40}$; and MS600, 70-80 kb from the pseudoautosomal (Xp/Yp) telomere ${ }^{41}$. Probes were acquired from the American Type Culture Collection, the UK Human Genome Mapping Project probe bank (originators John A.L. Armour, Ray White, Yusuke Nakamura), the Japanese Cancer Research Resources Bank (originator Y. Nakamura) (42 $^{4}$, and gifts from Jan Dumanski (Karolinska Hospital, Stockholm, Sweden), Alec J. Jeffreys and John A.L. Armour (Leicester University, Leicester, UK) ${ }^{26}$, Gillian Bates (Guy's Hospital, London, UK), Douglas R. Higgs (Oxford, UK), William R.A. Brown (University of Oxford, UK) $)^{30}$ and Gilles Vergnaud (Institut de Biologie, Nantes, France). Probes used for characterization of the 22q deletions are as described ${ }^{43}$.

DNA analysis. Venous blood samples were taken from affected individuals and both parents, and Epstein-Barr virus-transformed 
lymphoblast cultures established. DNA was extracted from cell lines and peripheral blood, by standard procedures ${ }^{44}$. Samples from both parents and the affected offspring were digested with an appropriate restriction endonuclease, separated by electrophoresis through $0.8 \%$ agarose gels, transferred to nylon membranes and hybridized to radioactively labelled probes ${ }^{44}$. In most cases digests were appropriate for more than one probe, and so filters could be rehybridized.

PFGE was carried out using a BioRad CHEF apparatus. DNA digested in $0.7 \%$ agarose blocks was analysed in $1 \%$ agarose gels run in $0.5 \times$ Tris-borate EDTA buffer for 60 hours at $110 \mathrm{~V}$ with an initial pulse time of $15 \mathrm{~s}$ and a final pulse time of $50 \mathrm{~s}$.

Characterization of the $22 \mathrm{q}$ deletions was by dosage and RFLP analysis $^{43}$.

Fluorescence in situ hybridization. FISH was performed on lymphoblastoid cells. Cosmids were labelled with biotin-11-dUTP bynick translation, and hybridized according to standard protocols $\mathrm{s}^{45}$. Conjugated probe $(100 \mathrm{ng})$ and competitor DNA $(1 \mu \mathrm{g})$ were used for each slide. Probes were detected by fluorescein isothiocyanateconjugated avidin. Chromosome-specific biotinylated $\alpha$-satellite was purchased from Oncor and used to confirm the identity of chromosomes.

Reverse chromosome painting. Metaphase chromosomes were sorted on a Becton Dickinson FACStar Plus as described ${ }^{46}$. In none of the three cases studied could the deleted chromosome be separated from its normal homologue, so both were labelled. Five hundred chromosomes were amplified with degenerate primers (DOP-PCR), and $5 \mu \mathrm{l}$ of the product was amplified again in the presence of biotin11-dUTP (refs 46,47). Lowest background signal was achieved by using $0.025 \mathrm{U}$ of Taq polymerase in a $100 \mu \mathrm{l}$ amplification reaction and by including $10 \%$ glycerol in the reaction mixture. Labelled chromosomes $(500 \mathrm{ng})$ with $10 \mu \mathrm{g}$ of competitor DNA were hybridized to normal male chromosomes, following the protocol described above for FISH.

Calculation of informativeness. The informativeness for each probe is derived from the seven possible parental mating types ${ }^{16}$ (note that this is not directly related to the heterozygosity). Mating types are classified according to the distribution of alleles and have different probabilities of detecting UPD, monosomy and trisomy ${ }^{16}$. The calculation of overall informativeness of the test for UPD includes the figures for both isodisomy and heterodisomy. The probability that a locus can detect each chromosomal abnormality is calculated by summing the informativeness for every mating type scored. In those cases where more than one locus per telomere was analysed, the most informative mating type for monosomy among those scored was used when summing the probabilities of detection. Informativeness for analysis of all chromosomes was derived ${ }^{16}$. with a value of $\psi=1 / 3$ applied for the calculation of the probability of detection of UPD.

\section{Acknowledgements}

We thank R. Boone, L. Kearney, S. Tosi, J. Armour, D. Higgs, J. Clegg and Sir David Weatherall for their help and contribution to this work. We would also like to acknowledge the help of the staff at Porton Down (in particular Sarah Andrews) for establishing cell lines and particularly Jan Dumanski for allowing us to use his $22 q$ cosmids. We thank John Clegg, John Old and Jeremy Martinson for making control samples available to us. We would also like to thank the families involved in this project for providing samples. This work was supported by the Wellcome Trust (J.F., A.O.M.W.) and the Medical Research Council of Great Britain (V.B.).

Received 22 September; accepted 21 December 1994. 
1. Rutter, M., Tizard, J. \& Whitmore, K. Education, Health and Behaviour (Longman, London, 1970).

2. Birch, H.G., Richardson, S.A., Baird, D., Horobin, G. \& Ilsley, R. Mental subnomality in the community: aclinical and epidemiologicalstudy. (Williams and Wilkins, Baltimore, 1970).

3. Drillien, C.M., Jameson, S. \& Wilkinson, E.M. Studies in mental handicap. Part I: Prevalence and distribution by clinical type and severity of defects. Arch. Dis. Childh. 41, 528-538 (1966).

4. McDonald, A.D. Severely retarded children in Quebec: prevalence, causes and care. Am. J. Men. Defic. 78, 205-215 (1973).

5. Gustavson, K.-H., Hagberg, B., Hagberg, G. \& Sars, K. Severe mental retardation in a Swedish county. II. Etiologic and pathogenetic aspects of children born 1959-1970. Neuropăediatrie 8, 293-304 (1977).

6. Laxova, R., Ridler, M.A.C. \& Bowen-Bravery, M. An etiological survey of the severely retarded Hertfordshire children who were born between January 1 , 1965 and December 31, 1967. Am. J. Med. Genet. 1, 75-86 (1977).

7. Elwood, J.H. \& Darragh, P.M. Severe mental handicap in Northern ireland. J. men. Defic. Res. 25, 147-155 (1981)

8. McQueen, P.C., Spence, M.W., Winsor, E.J.T., Garner, J.B. \& Pereira, L.H. Causal origins of major mental handicap in the Canadian maritime provinces. Dev. Med. and Child Neurol. 28, 697-707 (1986).

9. Hagberg, B., Hagberg, G., Lewerth, A. \& Lindberg, U. Mild mental retardation in Swedish school children -I. Prevalence. Acta paediat. scand. 70, 441-444 (1981).

10. Hagberg, B., Hagberg, G., Lewerth, A. \& Lindberg, U. Mild mental retardation in Swedish school children - II. Etiologic and pathogenetic aspects. Acta paediat. scand. 70, 445-452 (1981).

11. Einfeld, S.L Clinical assessment of 4500 developmentally delayed individuals. J. men. Defic. Res. 28, 129-142 (1984).

12. Broman, S., Nichols, P.L., Shaughnessy, P. \& Kennedy, W. Retardation in Young Children: A Developmental Study of Cognitive Deficit (Erlbaum, New Jersey, 1987).

13. Lamont, M.A. \& Dennis, N.R. Aetiology of mild mental retardation. Arch. Dis. Childh. 63, 1032-1038 (1988).

14. Bundey, S., Thake, A. \& Todd, J. The recurrence risks for mild idiopathic mental retardation. J. med. Genet. 26, 260-266 (1989)

15. Gostason, R., Wahlstrom, J., Johannisson, T. \& Holmqvist, D. Chromosomal aberrations in the mildly mentally retarded. J. men. Defic. Res. 35, 240-246 (1991).

16. Wilkie,A.O.M. Detection of cryptic chromosomal abnormalities in unexplained mental retardation: a general strategy using hypervariable subtelomeric DNA polymorphisms. Am. J. hum. Genet. 53, 688-701 (1993).

17. Lamb, J. et al. Detection of breakpoints in submicroscopic chromosomal translocation, illustrating an important mechanism for genetic disease. Lancet ii, 819-824 (1989).

18. Wilkie, A.O.M. et al. Clinical features and molecular analysis of the $\alpha$ thalassemia/mental retardation syndromes. I. Cases due to deletions involving chromosome band 16p13.3. Am. J. hum. Genet. 46, 1112-1126 (1990).

19. Altherr, M.R. etal. Molecular confirmation of Wolf-Hirschhorn syndrome with a subtle translocation of chromosome 4. Am. J. hum. Genet. 49, 1235-1242 (1991).

20. Goodship, J. et al. A submicroscopic translocation, $t(4 ; 10)$, responsible for recurrent Wolf-Hirschhorn syndrome identified by allele loss and fluorescent in situ hybridisation. J. med. Genet. 29, 451-454 (1992).

21. Kuwano, A., Ledbetter, S.A., Dobyns, W.B., Emanuel, B.S. and Ledbetter, D.H. Detection of deletions and cryptic translocations in Miller-Dieker syndrome by in situ hybridization. Am. J. hum. Genet. 49, 707-714 (1991).

22. Overhauser, J. et al. Prenatal diagnosis and carrier detection of a cryptic translocation by using DNA markers from the short arm of chromosome 5 . Am. J. hum. Genet. 45, 296-303 (1989).

23. Lamb, J. et al. De novo truncation of chromosome $16 \mathrm{p}$ and healing with (TTAGGG) in the $\alpha$-thalassemia/mental retardation syndrome (ATR-16). Am. J. hum. Genet. 52, 668-676 (1993).

24. Nicholls, R.D. Genomic imprinting and uniparental disomy in Angelman and Prader Willi syndromes: a review. Am. J. med.Genet. 46, 16-25 (1993).

25. Henry, I. et al. Somatic mosaicism for partial isodisomy in BeckwithWiedemann syndrome: a postfertilization event. Eur. J. hum. Genet. 1, 1929 (1993).

26. Armour, J.A.L., Povey, S., Jeremiah, S. \& Jeffreys, A.J. Systematic cloning of human minisatellites from ordered array charomid libraries. Genomics 8 , $501-512(1990)$.

27. Vergnaud, G. et al. The use of synthetic tandem repeats to isolate new VNTR loci. Cloning of a human hypermutable sequence. Genomics 11, 135-144 (1991).

28. NIH/CEPH Collaboratlve Mapping Group. A comprehensive genetic linkage map of the human genome. Science 258, 67-86 (1992).

29. Armour, J.A.L. Crosier, M. \& Jeffreys, A.J. A highly polymorphic minisatellite (pMS626) on chromosome 13 (D13S103). Nucl. Acids Res. 19, 5447 (1991).

30. Cooke, H.J., Brown, W.R.A. \& Rappold, G.A. Hypervariable telomeric sequences from the human sex chromosomes are pseudoautosomal. Nature 317, 687-692 (1985).

31. Armour, J.A.L., Crosier, M. \& Jeffreys, A.J. Human minisatellite alleles detectable only after PCR amplification. Genomics 12, 116-124 (1992).

32. Royle, N.J., Armour, J.A., Crosier, M. \& Jeffreys, A.J. Abnormal segregation of alleles in CEPH pedigree DNAs arising from allele loss in lymphoblastoid DNA. Genomics 15, 119-122 (1993).

33. James, R.S. et al. A systematic search for uniparental disomy in carriers of chromosomal transiocations. Eur. J. hum. Genet. 2, 83-95 (1994).

34. Hing, A.V., Helms, C. \& Donis-Keller, H. VNTR and microsatellite polymorphisms within the subtelomeric region of $7 \mathrm{q} . \mathrm{Am}$. J. hum. Genet. $\mathbf{5 3}$, 509-517 (1993).

35. Ledbetter, D.H. Minireview: Cryptic transiocations and telomere integrity. Am. J. hum. Genet. 52, 451-456 (1992).

36. Reed, P.W. et al. Chromosome-specific microsatellite sets for fluorescencebased, serni-automated genome mapping. Nature Genet. 7, 390-395 (1994).

37. Nakahori, Y. et al. Molecular heterogeneity of the fragile $X$ syndrome. Nucl. Acids Res. 19, 4355-4359 (1991).

38. Wilkie, A.O.M. et al. Stable length polymorphism of up to $260 \mathrm{~kb}$ at the tip of the short arm of human chromosome 16. Cell 64, 595-606 (1991).

39. Bucan, M. et al. Physical maps of $4 \mathrm{p} 16.3$, the area expected to contain the Huntington disease mutation. Genomics 6, 1-15 (1990).

40. Bates, G.P. et al. Defined physical limits of the Huntington disease gene candidate region. Am. J. hum. Genet. 49, 7-16 (†991).

41. Henke, A., Fischer, C. \& Rappold, G.A. Genetic map of the human pseudoautosomal region reveals a high rate of recombination in female meioses at the Xp telomere. Genomics 18, 478-485 (1993).

42. Takahashi, E.-i., Yamakawa, K., Nakamura, Y. \& Hori, T.-a. A high-resolution cytogenetic map of human chromosome 3: localization of 291 new cosmid markers by direct R-banding fluorescence in situ hybridization. Genomics markers by direct R-band

43. Nesslinger, N.J. et al. Clinical, cytogenetic, and molecular characterization of seven patients with deletions of chromosome 22q13.3. Am. J. hum. Genet. 54, 464-472 (1994).

44. Sambrook, J., Fritsch, E.F. \& Maniatis, T. Molecular Cloning: A Laboratory Manual. (2nd edn., New York, Cold Spring Harbor Laboratory Press, 1989).

45. Buckle, V.J. \& Rack, K. Fluorescent in situ hybridisation. in Human Genetic Diseases: A Practical Approach 2nd edn (ed. Davies, K.E.) 59-80 (IRL Press, Oxford, 1993).

46. Rack, K.A. et al. Characterization of three de novo derivative chromosomes 16 by 'reverse chromosome painting' and molecular analysis. Am. J. hum. Genet. 52, 987-997 (1993).

47. Telenius, $\mathrm{H}$. et al. Degenerate oligonucleotide-primed PCR: genera amplification of target DNA by a single degenerate primer. Genomics 13, $718-725(1992)$

48. MacDonald, M.E. et al. Clustering of multiallele DNA markers near the Huntington's disease gene. J. clin. Invest. 84, 1013-1016 (1989).

49. Wong, Z., Wilson, V., Patel, I., Povey, S. \& Jeffreys, A.J. Characterisation of a panel of highly variable minisatellites cloned from human DNA. Ann. hum. Genet. 51, 269-288 (1987).

50. Crosier, M., Armour, J.A.L. \& Jeffreys, A.J. A highly polymorphic minisatellite (pMS627) on chromosome 14 (D14S44). Nucl. Acids Res. 19, 5446 (1991).

51. Jarman, A.P., Nicholls, R.D., Weatherall, D.J., Clegg, J.B. \& Higgs, D.R Molecular characterization of a hypervariable region downstream of the human $\alpha$-globin gene cluster. EMBO J. 5,1857-1863 (1986).

52. Armour, J.A.L., Wong, Z., Wilson, V., Royle, N.J. \& Jeffreys, A.J. Sequences flanking the repeat arrays of human minisatellites: Association with tandem and dispersed repeat elements. Nucl. Acids Res. 17, 4925-4935 (1989). 